\title{
DENTAL LAW AND ETHICS COURSE
}

The first cohort of students has graduated from the University of Bedfordshire's unique Postgraduate Certificate in Dental Law and Ethics course (pictured right: M. Patel, G.Jaswal, H. Wassif [course lead], A. McDonald, D. Shaffer).

This course provides opportunity to critically examine dental care from relevant ethical, legal and professional standpoints. It is a part-time course that combines study days with supported online learning. Some examples of the topics covered include consent, negligence, human rights, whistleblowing and safeguarding.

The Bedfordshire certificate is the only university-accredited course in law and ethics specially designed for dental professionals.

A course taster day will take place on Friday 9 May 2014 and the full course starts in October 2014. For course enquiries contact: Ms Linda Ellingham; email linda. ellingham@beds.ac.uk or telephone 01582489074.

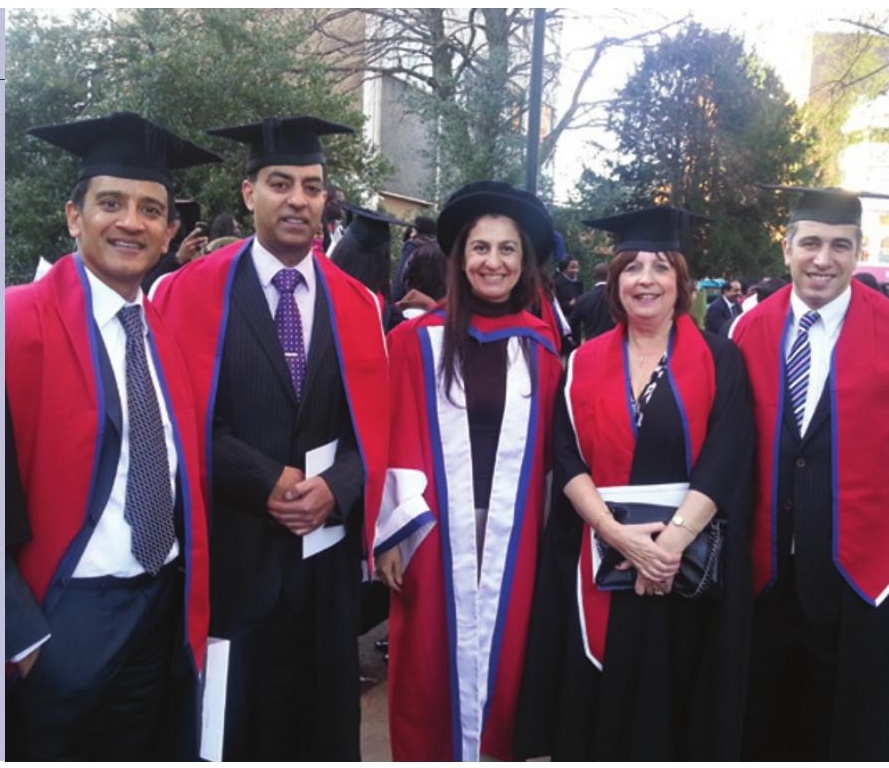

\section{MARRYING FORM AND FUNCTION}

The new A-dec 400 dental chair marries form and function for less complexity and more style, at a beautiful price.

The A-dec 400 is a truly ambidextrous package which can be configured to accommodate varied preferences. The delivery and support modules are able to quickly and easily rotate around the chair for complete left/right compatibility for the dental team.

Its features don't end there. It was designed to put your patients at ease with its double articulating headrest, toe board tilt and virtual pivot which synchronises with the movement of the chair and anatomy and motion of the patient.

You can count on A-dec products to work consistently so you can stay focused on what matters most: the patient. Like all A-dec dental chairs, the A-dec 400 is built with reliability in mind to keep your downtime to a minimum and is now offered with a five year warranty.

For more information call A-dec on 0800233285 or visit www.a-dec.co.uk.

\section{LEARN TO SPOT CANCER OVER LUNCH}

As official sponsors of the Mouth Cancer Foundation's (MCF) life-saving initiative, the Mouth Cancer Screening Accreditation Scheme, practice-branded dental plan provider DPAS is delighted to be offering a new 'Lunch \& Learn' session free to their client practices.

In promoting the importance of early detection to dental professionals, in turn helping to raise public awareness of the disease, MCF has been working with DPAS to devise these Lunch \& Learns, as well as training their regional Practice Consultants to raise awareness of the signs of mouth cancer and MCF's '2 Minutes to Save a Life' screening protocol.
Demonstrating the speed and simplicity of the screening process, DPAS is providing lunch and delivering one hour of external CPD to the whole dental team, which is required to engage in the scheme.

If you would like to book a 'Lunch \& Learn' in 2014, call DPAS on 01747870910.

For further information on the Mouth Cancer Foundation visit www.mouthcancerfoundation.org. www.dpas.co.uk
PRACTISE LASER DENTISTRY ON SHEEP

Quicklase sponsor the British Institute of Laser Dentistry, a non-profit organisation set up to provide laser hands-on courses for dentists. On the UK courses the president, Dr Anoop Maini, presents lasers in clinical application followed by an afternoon of 'hands-on'.

Quicklase lasers are used for the hands-on session; it is the most important part of the course so sheep heads are used to practise on. Over 25 procedures are executed in the hands-on sessions such as troughing for accurate impression and gingival re-contouring as well as pulpotomy, gum pigmentation and many more, not to be missed.

Laser assisted dentistry has modernised dentistry and has become a vital part of the industry. Furthermore, at the seminar hands-on, Quicklase always offer special deals for attendees.

Call 01227780009 to book or visit www.quicklase.com to watch the BILD hands-on video and see testimonials.

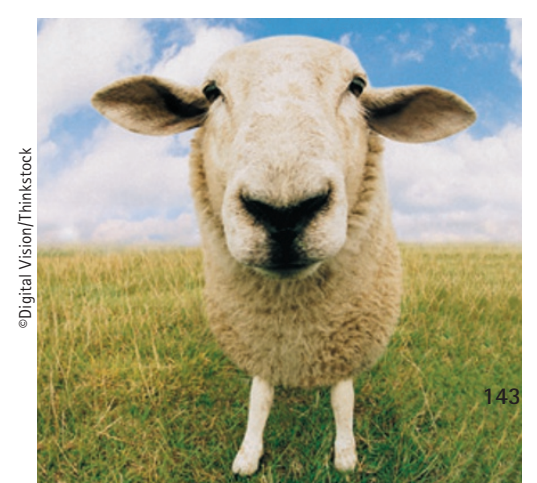

